Boletín de la Sociedad Zoológica del Uruguay, 2021

Vol. 30 (2): e30.2.7

ISSN 2393-6940

https://journal.szu.org.uy

DOI: https://doi.org/10.26462/30.2.7

\title{
EVALUACIÓN PRELIMINAR DE LA DIVERSIDAD DE ANFIBIOS EN LA ALAMEDA DE SAN CARLOS (MALDONADO, URUGUAY), A TRAVÉS DE UN MONITOREO ACÚSTICO PASIVO
}

\author{
Martina Fernández ${ }^{1}(\mathbb{D})$, Matilde Méndez ${ }^{1}(\mathbb{D})$, Bruno Agosto' ${ }^{1}$, Daniela Albornoz ${ }^{1}$ (D), \\ Francesca Cornelli' ${ }^{1}$, Eugenia Dutra' ${ }^{1}$, Sabrina González' ${ }^{1}$, María Moulia' ${ }^{1}$ (D) , Rodrigo Núñez ${ }^{1}(\mathbb{D})$, \\ Franco Teixeira de Mello ${ }^{2}$ (D), Lucía Ziegler ${ }^{2 *}$ (D) . \\ 1. Taller Interdisciplinario de Tópicos Regionales II. Licenciatura en Gestión Ambiental. Centro Universitario \\ Regional Este. Universidad de la República. Tacuarembó s/n, entre Av. Artigas y Aparicio Saravia, Maldonado \\ CP 20000, Uruguay. \\ 2. Departamento de Ecología y Gestión Ambiental. Centro Universitario Regional del Este. Universidad de la \\ República. Tacuarembó s/n, entre Av. Artigas y Aparicio Saravia, Maldonado CP 20000, Uruguay.
}

*Autor para correspondencia: lucia.ziegler@gmail.com

Fecha de recepción: 27 de junio de 2021

Fecha de aceptación: 27 de octubre de 2021

\section{RESUMEN}

El monitoreo de especies y comunidades es clave en la gestión de la conservación de la biodiversidad, la elaboración y la planificación de políticas de conservación. Los anfibios experimentan a nivel global una importante crisis de diversidad. En el caso de Uruguay se cuenta con un registro de 47 especies nativas, donde el $34 \%$ de ellas se encuentran en alguna categoría de amenaza. Este trabajo tuvo como objetivo aportar a la generación de información sobre la diversidad de anfibios a través de un monitoreo acústico pasivo en la Alameda de San Carlos. Durante este estudio se obtuvieron 511 grabaciones, con un total de 2555 minutos de grabación. Se registraron e identificaron un total de 14 especies de anfibios. Durante los 22 días de muestreo, fue notoria la variación temporal en el registro de especies, Boana pulchella y Scinax squalirostris fueron las únicas que estuvieron activas durante todo el muestreo, mientras que el resto de las especies lo hicieron de forma intermitente. Este estudio destaca por estar basado en el monitoreo acústico pasivo de los cantos de anfibios, aportando información inexistente para el área de estudio y mostrando un gran potencial en actividades de enseñanza además del científico.

Palabras Claves: Arroyo Maldonado, humedales, diversidad de anfibios, biomonitoreo

\section{ABSTRACT}

Preliminary assessment of the diversity of amphibians in the Alameda de San Carlos (Maldonado, Uruguay), through passive acoustic monitoring. Biological monitoring, from species to communities, is key to biodiversity conservation management, policy development and planning. Globally, amphibians are experiencing a major diversity crisis. In the case of Uruguay, there is a record of 47 native species, $34 \%$ of which have been assigned to some category of threat. The objective of this work was to contribute to the generation of information on amphibian diversity through passive acoustic monitoring in the Alameda de San Carlos. During this study, 511 recordings were obtained, with a total of 2555 minutes of recording. A total of 14 amphibian species were recorded and identified. During the 22 days of sampling, temporal variation in the activity of recorded species was notorious. Boana pulchella and Scinax squalirostris were the only ones that were active during the entire sampling, while the rest of the species were active intermittently. This study represents the first work based on passive acoustic monitoring of anuran calls in our country, providing novel information that was not available for the study area and showing great potential for teaching activities in addition to scientific ones.

Keywords: Maldonado Stream, wetlands, amphibian diversity, biomonitoring.

\section{INTRODUCCIÓN}

El monitoreo de especies y comunidades es clave en la gestión de la conservación de la biodiversidad y la elaboración y planificación de políticas al respecto. La información proporcionada por los monitoreos es básica para evaluar el estado de las poblaciones naturales, evaluar su dinámica y así poder estimar las 
tendencias y generar alertas tempranas sobre la disminución de poblaciones y/o extinción de las mismas. Por último, y no menos importante, donde existe una gestión activa de la biodiversidad, la información proveniente de los monitoreos permite evaluar la eficacia de las políticas de gestión (Lindenmayer et al., 2011).

Los anfibios experimentan a nivel global una importante crisis de diversidad, donde un elevado porcentaje de las especies conocidas se encuentra en alguna categoría de amenaza (Stuart et al., 2004). Existen diversas causas probadas que actúan de forma tanto individual como en sinergia, como ser el cambio climático, la destrucción de hábitats, tráfico de especies e introducción de especies exóticas y la aparición de enfermedades infecciosas emergentes (Hof, Araújo, Jetz y Rahbeck, 2011; Grant et al., 2016, Grant, Miller y Muths, 2020). Uruguay cuenta con una diversidad de anfibios de 48 especies registradas hasta el momento, compuesta por 47 especies nativas y una especie exótica invasora (Carreira y Maneyro 2015; Maneyro, Langone y Carreira, 2019; Pereyra et al., 2021). De acuerdo a los criterios de Listas Rojas de UICN (Canavero et al., 2010; Carreira y Maneyro, 2015), del total de especies un $34 \%$ (16 especies) se encuentran en alguna categoría de amenaza, mientras que cuatro especies presentan Datos Insuficientes (DD). Debido a la compleja situación de conservación de este grupo a nivel global, que se replica a escala nacional, en nuestro país resulta relevante incrementar el conocimiento sobre la ecología, distribución y amenazas de las especies de anfibios. En este sentido se ha avanzado mucho sobre la distribución de las diferentes especies (Núñez, Maneyro, Langone y De Sá, 2004; Grattarola et al., 2019, 2020). Sin embargo, es necesario seguir avanzando en los inventarios de especies en diferentes ambientes de nuestro país ya que aún persisten importantes vacíos de información (Grattarola et al., 2020). Según Canavero y colaboradores (2010a) solo el litoral del Río de la Plata, la costa atlántica, parte de la cuesta basáltica y el litoral norte, cuentan con suficiente información sobre la diversidad de anfibios como para considerarlas bien conocidas. Es importante destacar que gran parte de la información se ha obtenido de colecciones científicas basadas en registros históricos, por lo que no necesariamente representa la ocurrencia de las especies en la actualidad. En un artículo reciente, Grattarola y colaboradores (2019) hacen un análisis temporal de los registros pertenecientes a la clase Amphibia en Uruguay. Allí reportan que solo el $27 \%$ de los registros son de los últimos 30 años, por lo que la mayor parte de los registros sobre los que se hacen inferencias sobre los patrones de distribución no consideran el efecto de las presiones antrópicas y su efecto en las poblaciones locales de muchas especies (Canavero et al., 2010; Grattarola et al., 2019; Laufer et al., en prensa).
El estudio de anfibios en su ambiente incluye una diversidad de técnicas, siendo el uso de las mismas dependientes de los objetivos del estudio (Sutherland, 2006; Dodd, 2009). Entre los principales métodos utilizados en nuestro país se encuentran los métodos basados en la colecta manual o mediante el uso de trampas, métodos de observación directa (Maneyro, 2019) y basados en la detección acústica (Ziegler y Canavero, 2019). Los métodos basados en la detección mediante señales acústicas han tenido un fuerte desarrollo en las últimas dos décadas. En parte debido a que las tecnologías para el registro de señales acústicas se han hecho más accesibles, fiables y portátiles (Dorcas, Price, Walls y Barichivich, 2009; Ziegler y Canavero, 2019). Pero también debido a que estas técnicas permiten obtener información relevante sobre la historia natural y ecología de las especies sin ser invasivas, aspecto que cobra particular importancia cuando se trata de trabajar con especies raras y/o amenazadas (Ziegler y Canavero, 2019).

Existen básicamente dos grupos de técnicas para obtener datos sobre la biología de los anuros a través de sus vocalizaciones. Por un lado, las técnicas manuales o activas están constituidas por las grabaciones manuales, e incluyen los muestreos auditivos. Estas técnicas son las que tienen más representación en la literatura hasta el momento, dado que solo requieren del conocimiento de la identidad específica de las señales acústicas y se pueden llevar a cabo aun sin necesidad de soporte tecnológico (i.e. muestreos auditivos), o con técnicas y equipos de grabación simples (Sutherland, 2006). En este sentido, si bien se trata de una metodología muy eficiente y en muchos casos económica, implican un esfuerzo muy elevado de trabajo en campo por parte de especialistas o técnicos bien entrenados en el reconocimiento de las vocalizaciones (Ziegler y Canavero, 2019). Más recientemente se han hecho muy populares las técnicas pasivas de registro de señales (Dorcas et al., 2009). Éstas son cada vez más sofisticadas, pero, a la vez accesibles, permitiendo el monitoreo continuo, abarcando escalas temporales que hasta hace poco tiempo eran impensadas (Dorcas et al., 2009; Blumstein et al., 2011). Las ventajas de este método es que amplían las capacidades de monitoreo acústico al facilitar la posibilidad de hacer registros continuos en el tiempo, donde se pueden detectar los momentos de mayor actividad de las diferentes especies, y en el espacio pudiendo cubrir grandes áreas dependiendo del número de grabadores que se instalen. Además, es también cada vez más habitual que este tipo de información quede guardada en colecciones científicas digitales (Toledo et al., 2015; Dena, Rebouças, Augusto-Alves y Toledo, 2018; Meineke, Davies, Daru y Davies, 2019; Ziegler y Canavero, 2019).

El presente trabajo tiene como objetivo aportar a la generación de información sobre la presencia de 


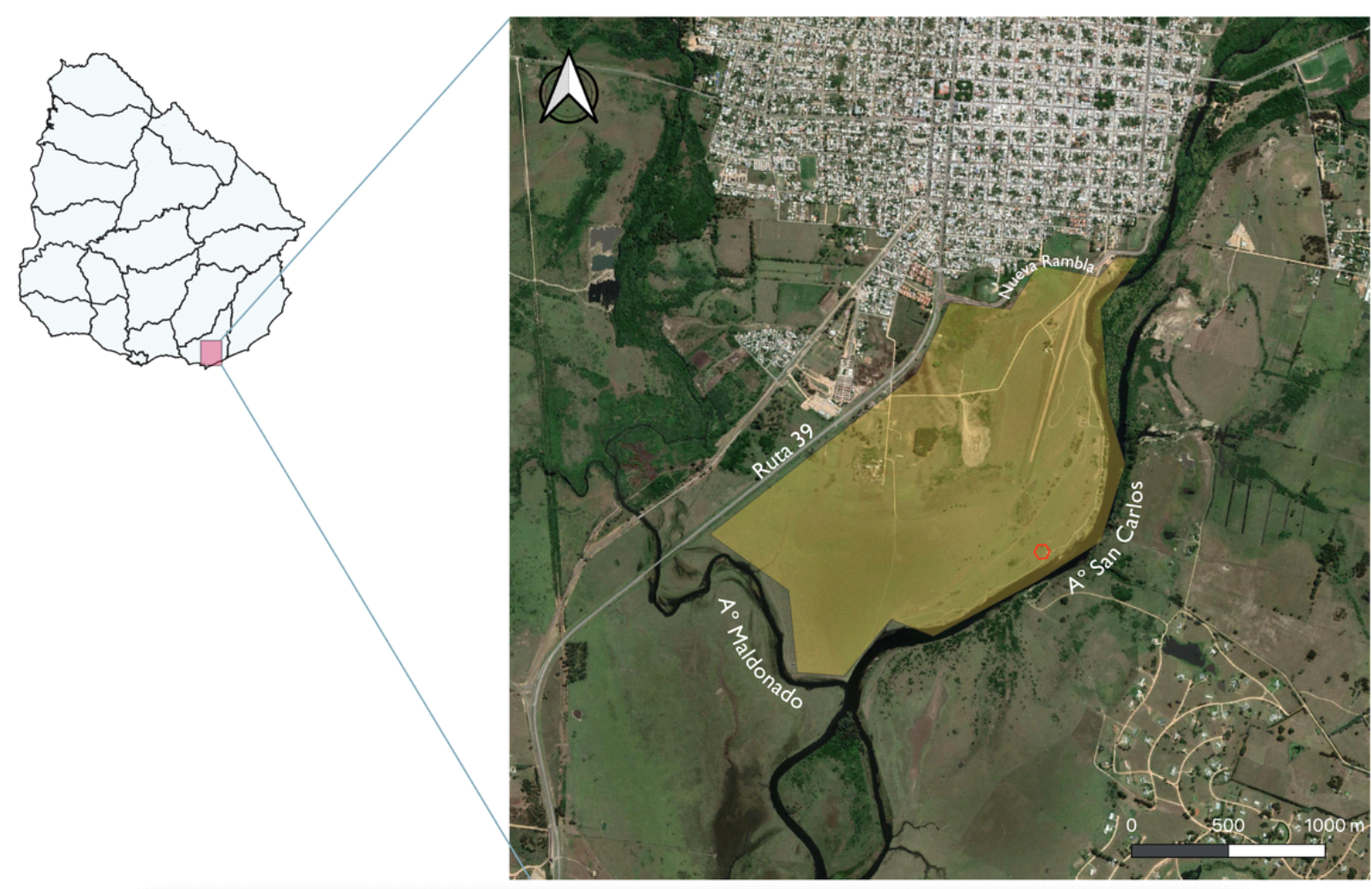

Fig. 1. Mapa e imagen satelital del sitio de muestreo. En verde claro se demarca la Alameda de San Carlos, delimitada por la Ruta 39 y los Arroyos Maldonado y San Carlos. Dentro del área de estudio se marca el sitio (círculo rojo) donde estaba colocada la unidad de registro acústico atomática.

especies de anfibios a través de un monitoreo acústico pasivo en la Alameda de San Carlos (Maldonado, Uruguay), en un sitio sin previos registros de su anurofauna. Se presenta asimismo un análisis del patrón de actividad de las especies más abundantes. Este trabajo fue realizado por estudiantes en el marco del curso Taller Interdisciplinario de Tópicos Regionales II de la Licenciatura en Gestión Ambiental.

\section{MATERIALES Y MÉTODOS}

\section{Área de estudio}

El área de estudio, conocida como La Alameda, está ubicada al sur de la Ciudad de San Carlos, Departamento de Maldonado (Uruguay). Está delimitada por los arroyos Maldonado (SW), San Carlos (E-SE) y por la Ruta Nacional 39 (W-NW) y la Rambla Este (perimetral) de San Carlos al norte (Fig. 1), abarcando un área total de 228 há. El área pertenece a la cuenca baja del Arroyo Maldonado, y representa la zona de humedales dulceacuícolas más al sur de la cuenca. Se trata de una zona compuesta principalmente por humedales formados por zonas de pradera inundable y charcos temporales y permanentes; éstos constituyen la planicie de inundación de los arroyos antes mencionados. Parte del área presenta también pastizales, así como parches de bosque mixto, con presencia de especies tanto nativas como exóticas.

La diversidad de anfibios fue evaluada mediante un monitoreo acústico pasivo entre los meses de setiembre y octubre de 2020. Para esto se seleccionó un charco en la zona media del área de estudio. El grabador se colocó adosado a un árbol en un parche de bosque contiguo al charco. Este charco se encontraba en una matriz de pastizal y a pocos metros del cauce del Arroyo San Carlos (ver Fig. 1). Para los registros acústicos se utilizó una Unidad Autónoma de Registro (ARU por sus siglas en inglés) Audiomoth ( O p e n A o u s t i c D e i c e s, https://www.openacousticdevices.info). La misma se programó para grabar los primeros 5 minutos de cada hora del día, y estuvo activa en el sitio de estudio durante 22 días, entre el 16 de setiembre y el 7 de octubre de 2020. Posteriormente se identificaron las especies a través de la escucha directa y visualización de las grabaciones (Ziegler y Canavero, 2019), utilizando el software Audacity (v 2.4.2, Audacity Team 2018).

Los cantos de los anuros fueron identificados al nivel de especie utilizando grabaciones de referencia y 


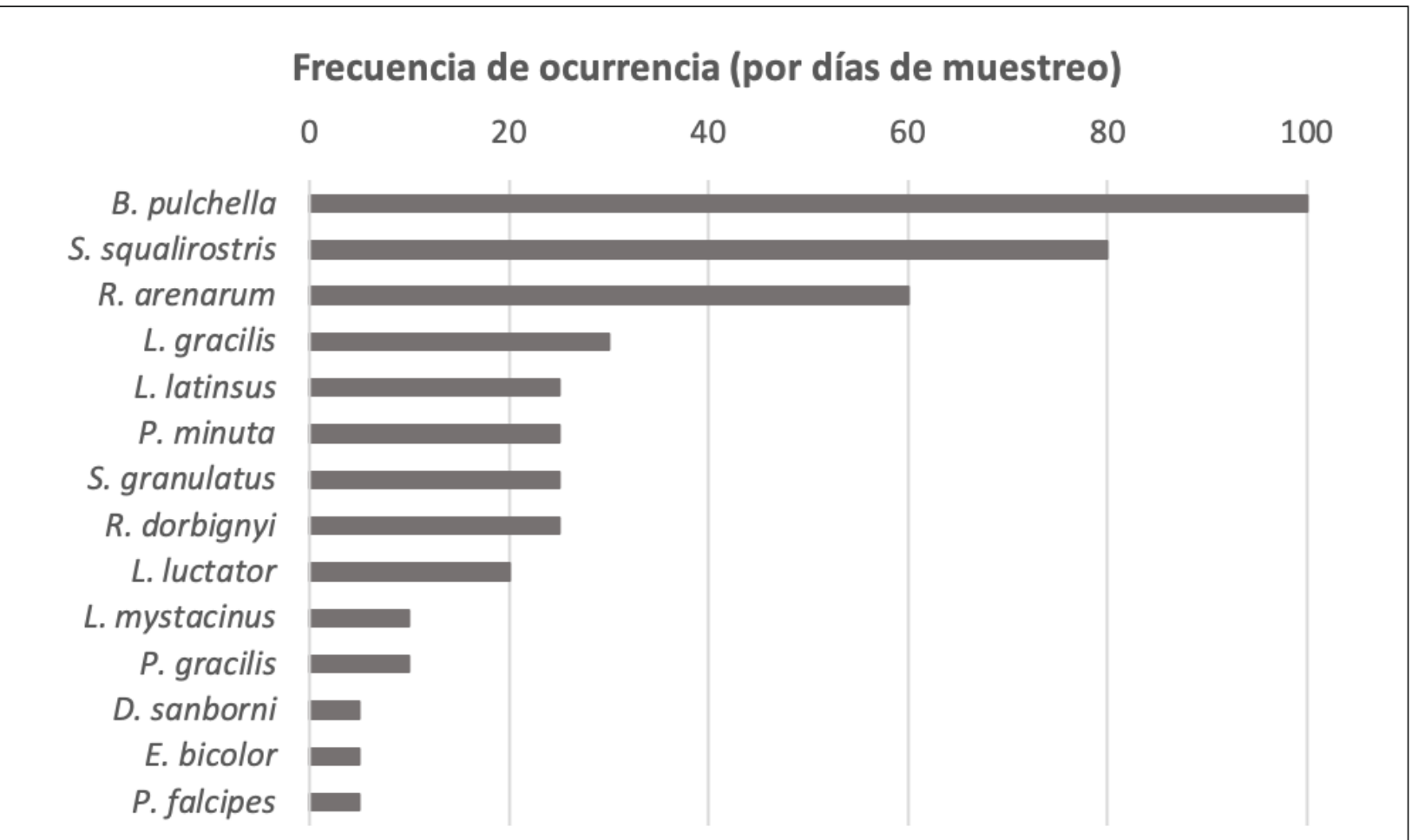

Fig. 2. Frecuencia de ocurrencia de las 14 especies de anfibio registradas para los 22 días de muestreo.

corroborados posteriormente por uno de los autores (LZ). Con los datos obtenidos se construyó una matriz de presencia/ausencia en base a la actividad acústica de cada una de las especies registradas (presencia = al menos un individuo de esa especie se registró cantando en esa grabación; ausencia = no se registró ningún individuo de esa especie cantando en esa grabación), para cada hora del día durante los 22 días de registro. El nivel de actividad de cada especie fue cuantificado en base a la frecuencia de ocurrencia por hora del día, durante todo el período de muestreo.

\section{RESULTADOS}

Durante este estudio, a través del dispositivo de registro acústico pasivo se obtuvieron 511 grabaciones, con un total de 2555 minutos de grabación de sonidos. Se registraron e identificaron un total de 14 especies de anfibios. Estas fueron Rhinella arenarum (Hensel, 1875), R. dorbignyi (Dumeril \& Bibron, 1841), Boana pulchella (Dumeril \& Bibron, 1841), Scinax granulatus (Peters, 1872), S. squalirostris (Lutz, 1925), Dendropsophus sanborni (Schmidt, 1944), Pseudis minuta (Günther, 1858), Leptodactylus gracilis (Dumeril \& Bibron, 1841), L. latinasus (Jimenez de la Espada, 1875), L. luctator (Hudson, 1892), L. mystacinus (Burmeister, 1861), Physalaemus gracilis (Boulenger,
1883), Pseudopaludicola falcipes (Hensel, 1875), y Elachistocleis bicolor (Guerin-Meneville, 1838). Durante los 22 días que duró el muestreo, fue notoria la variación temporal en el registro de especies, estando algunas pocas activas durante todo el muestreo (e.g. Boana pulchella, Scinax squalirostris, ver Fig. 2), mientras que la mayor parte de las especies estuvieron activas de forma intermitente. Tres de las especies registradas lo fueron hacia el final del muestreo $(D$. sanborni, E. bicolor y L. mystacinus), que además fueron las especies con un único evento de registro.

Por otra parte, las especies también presentaron patrones de actividad diarios idiosincráticos. Si bien fueron pocas las especies con una actividad de canto persistente, se pudieron identificar especies típicamente nocturnas (e.g. B. pulchella, P. minuta, $P$. gracilis; ver Fig. 3 a, d y h), y especies cuya actividad parece ser independiente de la hora del día (e.g. $S$. squalirostris, $R$. arenarum; ver Fig. $3 \mathrm{c} \mathrm{y} \mathrm{e).} \mathrm{Por} \mathrm{el}$ contrario, en este estudio no se encontraron especies típicamente diurnas.

\section{DISCUSIÓNY CONCLUSIONES}

En el presente trabajo se presenta la primera lista de especies de anfibios para el área de estudio y sus alrededores. La metodología permitió obtener datos 
5

Diversidad de anfibios en la Alameda de San Carlos

a)

B. pulchella

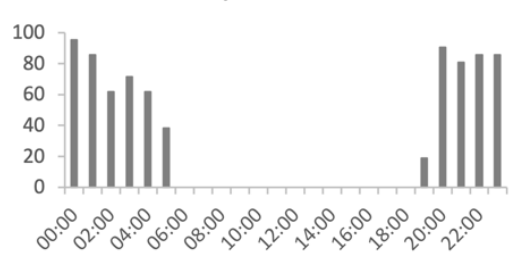

c)

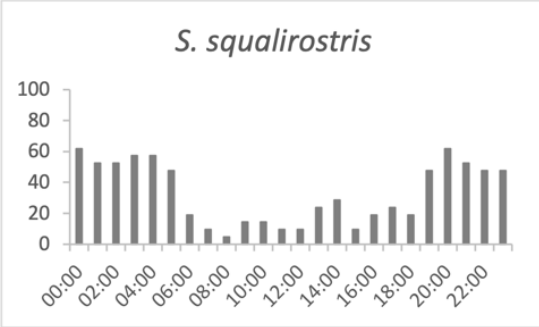

e)

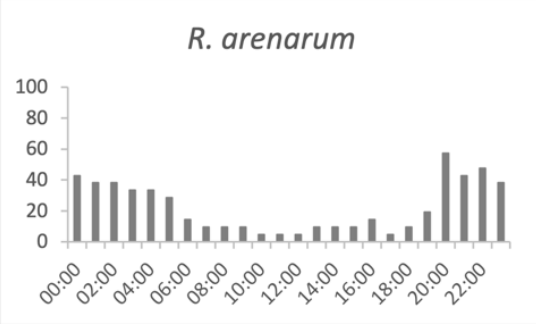

g)

L. gracilis

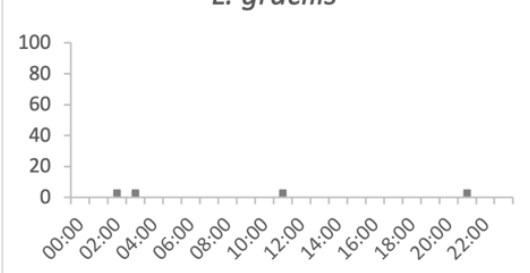

i)

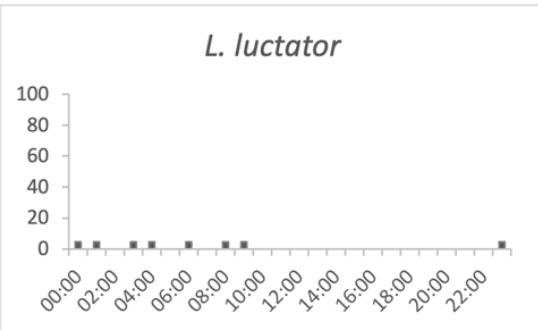

k)

E. bicolor

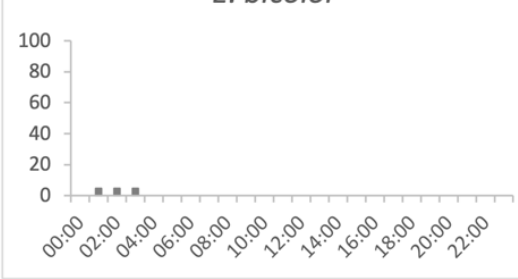

b)

S. granulatus

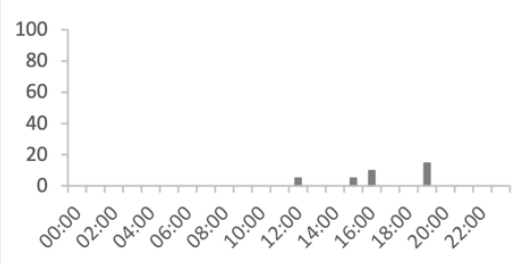

d)

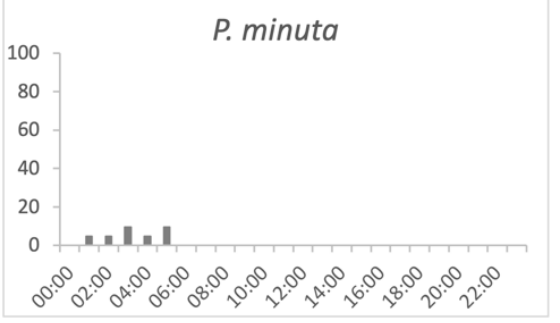

f)

R. dorbignyi

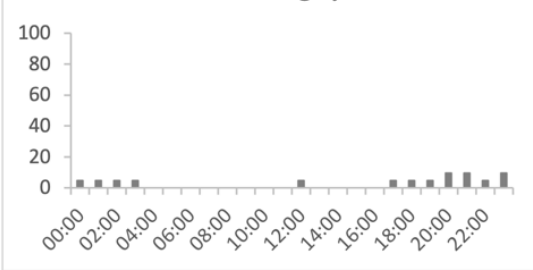

h)

L. latinasus

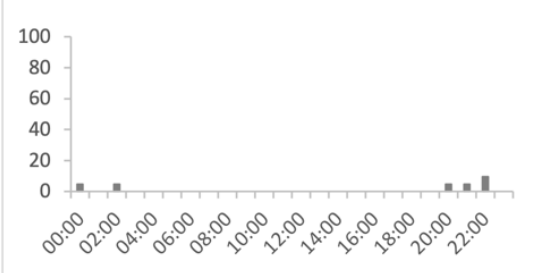

j)

P. gracilis

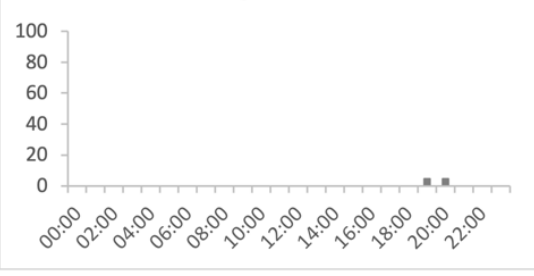

Fig. 3. Patrón diario de actividad de 11 de las 14 especies registradas en el sitio de estudio. El eje $Y$ representa la frecuencia de ocurrencia para cada hora del día, para el total de días del estudio $(\mathrm{n}=22)$. No se incluyen Scinax squalirostris, Leptodactylus mystacinus y Pseudopaludicola falcipes, especies para las cuales hay un único registro.

Bol. Soc. Zool. Uruguay (2a época). 2021. Vol. 30 (2): e30.2.7 ISSN 2393-6940

(c) (i) 
durante las 24 horas del día, en un período de 22 días consecutivos obteniéndose una lista de 14 especies. Si bien la lista de especies registradas no presenta especies de particular interés para su conservación, ni agrega especies a lo ya registrado para la zona a partir de registros en colecciones o reportes puntuales para el área de San Carlos y adyecencias (Grattarola et al., 2019), se trata del primer estudio sistemático de la diversidad de anfibios de La Alameda de San Carlos. Cabe destacar que dado lo restringido del muestreo en términos temporales, es posible que se trate de una lista parcial y que la diversidad local de anfibios sea aún mayor. De hecho, es notoria la ausencia de algunas especies comunes y abundantes en todo el territorio, como es el caso del escuerzo chico, Odontophrynus americanus. Esta y otras ausencias en la lista aquí presentada posiblemente se deban a fuerte estacionalidad y/o dependencia de factores abióticos en la actividad de muchas especies de anfibios.

Esta área a nivel departamental ha sido resaltada por diversos actores -sociales, políticos y académicosen los últimos años como sitio de importancia para su conservación (Caporale, Silvera, Lemos Zito, Baeza y Rodriguez, 2015; Caporale y Sciandro, 2015). De particular relevancia para el área es el Decreto Departamental 3931/2015 "De la creacion del Eco Parque Metropolitano del Humedal del Arroyo Maldonado" como reserva departamental. El objetivo de la creación de este Parque es "es preservar el ecosistema y poner en valor la biodiversidad". En este contexto, y considerando en particular el Art. 2 de dicho decreto en su mención del desarrollo de la dimensión Biológica-Ecológica como objetivo específico ("Promover el conocimiento público de los valores ecosistémicos del predio y el compromiso participativo tendiente a su preservación"), el presente trabajo representa un claro aporte en este sentido, sumándose a otros esfuerzos recientes que desde la academia se vienen haciendo para revalorizar el área (Caporale y Sciandro, 2015; Caporale et al., 2015, Teixeira de Mello et al., 2021).

Si bien no existen otros trabajos que reporten la diversidad de anfibios en el área, la comparación con otros estudios en áreas cercanas permite resaltar lo eficiente de esta metodología en cuanto a la detección de especies presentes. Por ejemplo, en un estudio llevado adelante en el Balneario Solís, Dpto. de Maldonado (Canavero et al., 2008) se encontraron en total 10 especies de anuros, cuatro especies menos que las reportadas en el presente trabajo. Esta diferencia es particularmente notable dado que el mencionado estudio se extendió por más de un año, mientras que el presente estudio estuvo fuertemente acotado en el tiempo.

Este estudio resalta por su metodología basada en el monitoreo acústico pasivo de los cantos de anfibios. Si bien es una metodología que ya tiene dos décadas de uso cada vez más extendido en el monitoreo de la diversidad (Dorcas et al., 2009, Ziegler y Canavero, 2019), hasta el momento los autores no tienen conocimiento de publicaciones arbitradas que hayan utilizado esta metodología para conocer y reportar la diversidad de algún grupo biológico a nivel local (pero ver Cerruti 2018 para un ejemplo de uso de data logging acústico para una tesina de grado). En este sentido, aunque el abordaje manual de procesamiento de la información fue posible dado lo relativamente acotado del estudio y la amplia disponibilidad de procesamiento por haber sido la información recogida en el contexto de un curso previo entrenamiento (2555 minutos de registro / nueve estudiantes $<5$ horas de escucha por estudiante) y el chequeo posterior por parte de un experto (LZ). De esta forma, este tipo de abordaje se convierte en un método efectivo para la enseñanaza universitaria de herramientas de monitoreo de diversidad alternativas, a la vez que permite generar información puntual pero que representa un aporte efectivo al conocimiento y registro de la fauna local. Por último, cabe destacar el potencial de la información obtenida en este tipo de estudio.

Contar con el respaldo de grabaciones nos permite repetir y chequear la identificación de las especies, a diferencia de lo que puede ocurrir con la escucha directa en campo que se requiere contar con un fuerte conocimiento y entrenamiento previo. Además del grupo biológico de interés en este trabajo, a través del registro acústico pasivo se guarda a la vez información de otros grupos (e.g. aves, invertebrados) o incluso de usos y actividades humanas que se llevan a cabo en el área que pueden ser la base de otros estudios en el área. Este aspecto también muestra el potencial de la inclusión de esta metodología no solo en investigaciones académicas, sino también a través programas de monitoreo participativo o ciencia ciudadana (Sugai, Silva, Ribeiro Jr. y Llusia, 2019).

\section{AGRADECIMIENTOS}

Este trabajo fue en parte financiado por la Licenciatura en Gestión Ambiental en el marco del curso Taller Interdisciplinario de Tópicos Regionales II edición 2020. FTM y LZ son apoyados por el Programa de Desarrollo de las Ciencias Básicas (PEDECIBA, Biología y Geociencias) y por el Sistema Nacional de Investigadores (SNI).

Este trabajo ha sido realizado en memoria de nuestro colega, amigo y docente de la Lic. en Gestión Ambiental Juan María Clemente (el Checho).

\section{BIBLIOGRAFÍA}

Blumstein, D.T., Mennill, D.J., Clemins, P., Girod, L., Yao, K., Patricelli, G., ... Kirschel, A.N.G. (2011). Acoustic monitoring in terrestrial environments 
using micro- phone arrays: applications, technological considerations and prospectus. Journal of Applied Ecology, 48(3), 758-767.

Canavero, A., Arim, M., Naya, D.E., Camargo, A., da Rosa, I. y Maneyro, R. (2008). Calling activity patterns in an anuran assemblage: the role of seasonal trends and weather determinants. North-Western Journal of Zoology, 4, 29v41.

Canavero, A., Brazeiro, A., Camargo, A., da Rosa, I., Maneyro, R., y Núñez, D. (2010). Amphibian diversity of Uruguay: Background knowledge, inventory completeness and sampling coverage. Boletín de la Sociedad Zoológica de Uruguay, 19, 2-19.

Canavero, A., Carreira, S., Langone, J.A., Achaval, F., Borteiro, C., Camargo, A., ... Ziegler, L. (2010). Conservation status assessment of the amphibians and reptiles of Uruguay. Iheringia. Série Zoologia, 100(1), 05-12.

Caporale, M., y Sciandro, J.L. (2015). Plan de manejo del Ecoparque del humedal del $\mathrm{A}^{\circ}$ Maldonado, Uruguay: una oportunidad para la gestión integrada del patrimonio arqueológico costero. En: M. Scherer, y S.L. Rodríguez (Comp.). La Gestión Integrada de las Zonas Costeras y Marinas en Iberoamérica: Experiencias y Retos (p. 27). Simposio 6. XVI Senalmar-XVI Colacmar. Santa Marta DTHC. Colombia.

Caporale, M., Silvera, M., Lemos Zito, J., Baeza, J., y Rodriguez, O. (2015). Revalorizacion del patrimonio arqueológico del ecoparque del humedal del Arroyo Maldonado (Depto. Maldonado-Uruguay). Cuadernos del Instituto Nacional de Antropología y Pensamiento Latinoamericano-Series Especiales, 2(3), 163-176.

Carreira, S., y Maneyro, R. (2015). Lista roja de los anfibios y reptiles del Uruguay. Una evaluación del estado de conservación de la herpetofauna de Uruguay sobre la base de los criterios de la Unión Internacional para la Conservación de la Naturaleza. Dirección Nacional de Medio Ambiente, Montevideo.

Dena, S., Rebouças, R., Augusto-Alves, G., y Toledo, L.F. (2018). Lessons from recordings lost in Brazil fire: deposit and back up. Nature, 563(7732), 473-474.

Dodd, C.K. (2009). Amphibian ecology and conservation: a handbook of techniques. Oxford University Press.

Dorcas, M.E., Price, S.J., Walls, S.C., y Barichivich, W.J. (2009). Auditory monitoring of anuran populations. En: C.K. Dodd (Ed.) Amphibian ecology and conservation: a hand book of techniques (pp. 281-298). Oxford University Press, Oxford.

Grant, E.H.C., Miller, D.A.W., Schmidt, B.R., Adams, M.J., Amburgey, S.M, Chambert, T., ... Muths, E. (2016). Quantitative evidence for the effects of multiple drivers on continental-scale amphibian declines. Scientific reports, 6(1), 1-9.

Grant, E.H.C., Miller, D.A., y Muths, E. (2020). A Synthesis of Evidence of Drivers of Amphibian Declines. Herpetologica, 76(2), 101-107.

Grattarola, F., Botto, G., da Rosa, I., Gobel, N., González, E.M., González, J., ... PincheiraDonoso, D. (2019). Biodiversidata: An OpenAccess Biodiversity Database for Uruguay. Biodiversity data journal, 7, e36226.

Grattarola, F., Martínez-Lanfranco, J.A., Botto, G., Naya, D.E., Maneyro, R., Mai, P., ... PincheiraDonoso, D. (2020). Multiple forms of hotspots of tetrapod biodiversity and the challenges of open-access data scarcity. Scientific reports, 10(1), 1-15.

Hof, C., Araújo, M.B., Jetz, W., y Rahbek C. (2011). Additive threats from pathogens, climate and land-use change for global amphibian diversity. Nature, 480(7378), 516-519.

Laufer, G., Gobel, N., Kacevas, N., Lado, N., Cortizas, S., Carabio, M., ... Kolenc, F. (2021). Updating the distributions of four Uruguayan hylids (Anura: Hylidae): recent expansions or lack of sampling efforts?. Amphibian and Reptile Conservation, en prensa.

Lindenmayer, D.B., Gibbons, P., Bourke, M., Burgman, M., Dickman, C.R., Ferrier, S., ... Zerger, A. (2012). Improving biodiversity monitoring. Austral Ecology, 37, 285-294.

Maneyro, R. (2019). Colecta y manejo de anfibios en el ambiente. En: F. Teixeira de Mello (Ed.). Experimentación con animales no tradicionales en Uruguay (pp. 71-79). Universidad de la República, Comisión Honoraria de Experimentación Animal. Montevideo.

Maneyro, R., Langone, J. y Carreira, S. (2019). Anfibios. En: S. Carreira y R. Maneyro (Eds.). Libro Rojo de los Anfibios y Reptiles del Uruguay. Biología y conservación de los Anfibios y Reptiles en peligro de extinción a nivel nacional (pp. 17-106). DINAMA, Montevideo.

Meineke, E.K., Davies, T.J., Daru, B.H., y Davis, C.C. (2019). Biological collections for understanding biodiversity in the Anthropocene. Philosophical Transactions of the Royal Society B, Biological Sciences, 374, 20170386.

Núñez, D., Maneyro, R., Langone, J., y De Sá, R.O. (2004). Distribucion geografica de la fauna de anfibios del Uruguay. Smithsonian Herpetological Information Service, 134,1-36.

Pereyra, M.O., Blotto B.L., Baldo D., Chaparro J.C., Ron S.R., Elias-Costa A.J., ... Faivovich, J. (2021). Evolution in the genus Rhinella: A total evidence phylogenetic analysis of Neotropical true toads (Anura: Bufonidae). Bulletin of the American Museum of Natural History, 
447(1),1-156.

Stuart, S.N., Chanson, J.S., Cox, N.A., Young, B.E., Rodrigues, A.S., Fischman, D.L., y Waller, R.W. (2004). Status and trends of amphibian declines and extinctions worldwide. Science, 306(5702), 1783-1786.

Sutherland, W.J. (Ed.). (2006). Ecological census techniques: a handbook. Cambridge University Press.

Sugai, L.S.M., Silva, T.S.F., Ribeiro Jr., J.W., y Llusia, D. (2019). Terrestrial passive acoustic monitoring: review and perspectives. BioScience, 69(1), 15-25.

Teixeira de Mello, F., Canavero, A., Ríos, N., García, G., Suárez, B., Lozoya, J.P. y Loureiro, M. (2021) An isolated population of Austrolebias charrua (Rivulidae, Cyprinodontiformes) detected in a fragile ecosystem (Maldonado, Uruguay). Aquatic Conservation: Marine and Freshwater Ecosystems, doi: 10.1002/aqc.3727

Toledo, L.F., Tipp, C., y Márquez, R. (2015). The value of audiovisual archives. Science, 347, 484.

Ziegler, L., y Canavero, A. (2019). Métodos alternativos para el estudio de anfibios en campo: bioacústica en anuros desde los organismos a las comunidades. En: F. Teixeira de Mello (Ed.). Experimentación con animales no tradicionales en Uruguay (pp. 80-91). Universidad de la República, Comisión Honoraria de Experimentación Animal. Montevideo.

Editor de Sección: Raúl Maneyro 